\title{
Assessment of creeping discharge initiated by metal particles on the silicone rubber/XLPE interface in cable joints
}

\author{
Wen-Zhi Chang, Fei Du, Jian-Gang Bi, \\ Shuai Yuan, Yan-Peng Gong, Yuan Yang
}

\begin{abstract}
Creeping breakdown caused by metal particles on the silicone rubber/XLPE interface of prefabricated cable joints often happens. Study on the development process of such insulation defect-led partial discharge (PD) can serve as a basis for the assessment of its severity level. In this paper, metal particles are sandwiched on the rubber/XLPE interface of a $35 \mathrm{kV}$ prefabricated cable joint to simulate a creeping defect.
\end{abstract}

K e y w o r d s: cable insulation, power cable connecting, interface phenomena, silicone rubber, cross linked polyethylene insulation

\section{Introduction}

In order to keep the pressure, prefabricated cable joints are tightly coated on cable insulator, which forms the composite interface of silicon rubber and crosslinked polyethylene (XLPE). Statistics indicates that the composite interface is the weakness of the insulation, about $97 \%$ of the total number of faults in the cable joints are the creeping discharge [1-2]. In addition, the prefabricated cable joints need to be installed in the field condition, metal particles are easily left which leads to insulating deterioration. It is conducive to remove the insulation failure and enhance the reliability of the cable by studying the discharge process on the composite interface and by establishing the evaluation method of severity.

In [3-6], acoustic-electric pulse method was used to analysis the distribution of space charge, and then the quantitative calculation formula of interface charge was carried out. The instability of space charge in high electric field was the sign of silicon rubber breakdown. The flashover characteristics was also studied according to aggregation state of XLPE, and control of the aggregation state of semi crystalline polymers was used to enhance the insulation level. In [7-10], the influences of light, carbonization and electric mark caused by pressure, roughness, lacerations and conductive particles were studied, the estimation of lifetime was conducted under electric field stresses and moisture condition. Instead of using established combinatorial ageing models, considering thermal, mechanical and electrical stresses, the works considered variation in single stress factors, based on which decisions and forecasting methods were proposed for cable's protection. In [11], the aluminum foils were rapped onto the cable sheath at insulated cable joint to form coupling capacitance. PD patterns were found to depend upon the defect type varying with aging time. The study indicated the mechanism of degradation process leading to breakdown by comparing experimental results of XLPE cable joint and simulated XLPE cable joint. In [12], PD patterns of cable joints indicated that the existence of the defect inside the joint PD risks, was due to the obvious result of making bad process, meanwhile the oscillation detection technology effectively judged entirely correct. In [13], a metallic spike defect within a three-phase $11 \mathrm{kV}$ cable joint was made, which investigated the impact of single phase energization on PD activity, but was not further used in high voltage plant. In [14-18], the typical defects in power cable such as protrusion defect of cable joint, void defect, interface filled with silicone oil, interface without silicone oil, the defect led to treeing breakdown, and the interface with conductive path in main insulation were designed, then PD patterns of each defect were discussed based on statistical calculation, and PD inception voltage and breakdown voltage were studied which indicated that the effect on interface silicone oil might be thought to be similar phenomena against discharge in oil impregnated insulation systems. In [19-20], the three-dimensional models were established to analyze the influences of interface roughness, scratch and humidity on the electric field distribution of the composite interface of cable joints. The simulating analysis provides a theoretical basis for further research on the surface discharge along the composite interface, but the characteristics of PD with typical defects were still unsolved.

In summary, the composite interface in cable joint is not thoroughly studied. Researchers mainly aimed on the space charge, electric mark and properties of materials on

* China Electric Power Research Institute. Xiaoyingdong Road 15, Beijing, 100192, China, dufei@epri.sgcc.com.cn 

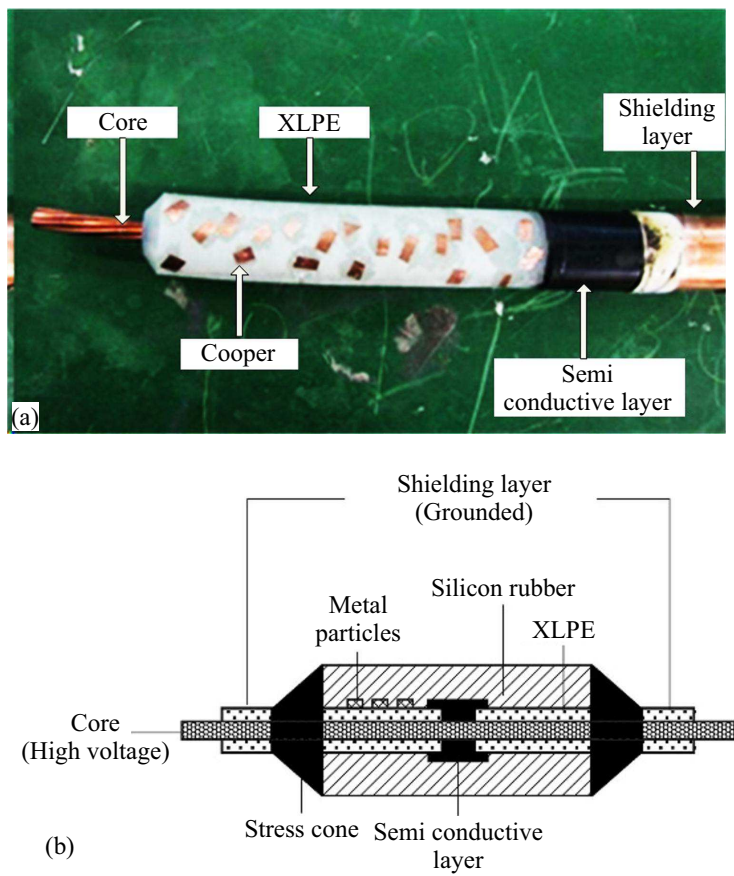

Fig. 1. Metal particles defect on the silicone rubber/XLPE interface:(a) - cooper on the Surface of XLPE, (b) - diagram of the defect sample

the surface of silicon rubber and XLPE, which could not achieve obtaining the insulation information in real time, and were inadequate in early flashover diagnosis. As the creeping discharge is caused by streamer, and develops in the form of $\mathrm{PD}$, the process of $\mathrm{PD}$ is employed to effective assess the insulation state, to prevent of creeping discharge as well.

In this work, the applied voltage on the test object was raised step by step to initiate the partial discharge and accelerate the degradation of the failure, where PD signals in the whole process are all measured. PD average energy, whole energy and PD number per unit time are chosen as the characterization parameters. The developing trends of these characterization parameters versus discharge time are then obtained. According to the developing trends, the entire developing process of PD is classified into four developing stages. For each stage, two-dimensional histograms of $\varphi$-n and $\varphi$ - $q_{\text {ave }}$ relationships as well as the grey-scale maps are generated, from which the phase distribution of $\mathrm{PD}$ repetition rate and PD average amount can be observed. Two features for the PD process are extracted from these-phase characteristic spectra. The experiment results show that visual differences between the phase characteristics spectra of each stage exist. The extracted features monotonically increase as the PD aggravates, and the growth rate increases in the late PD development. Based on the morphology of phase characteristic spectra and the variation of each PD development stage, the severity of creeping discharge arising from metal particle defect on silicone rubber/XLPE interface can be assessed.

\section{Experimental System}

\subsection{Defect model for PD test}

To reproduce the signals caused by the creeping discharge in cable joint, the real $35 \mathrm{kV}$ power cable and prefabricated cable joints are used as test sample. Before the joints are assembled, the semi conductive layer and metal shielding layer at the connection points of cables should be removed and XLPE is exposed. Some irregular pieces of copper with $5 \mathrm{~mm}-10 \mathrm{~mm}$ long and $0.05 \mathrm{~mm}$ thick are used as shown in Fig. 1(a). Silicone grease is smeared evenly on the surface of XLPE, then the prefabricated cable joints are assembled to connect the power cable which works as the metal particles defect, as shown in Fig. 1(b).

As the cooper is very thin, the pressure on the interface is high enough to keep the air gap nothingness, which namely ensures the defect as a single-typed metal particles defect.

The defect model originates in the real physical problem that the cable joint is introduced into the metal impurity during production process because of the construction technology. The volume and number of metal particles corresponding to actual defects are random. This study aims on the PD signals' development and evolution law during the whole breakdown process from the initial of PD to the final insulation breakdown based on this kind of defect. The most ideal test method is using a cable joint with actual defects, meanwhile, on the one hand, the test samples with actual defects are hard to obtain, on the other hand, the whole breakdown procedure can not be reappeared with the applied voltage and current in the laboratory conditions. Therefore, the defect model for PD test is designed and made based on the real principle of the metal particles defect. Compared with the actual defects, the severity of the defect in this paper is artificially aggravated to obtain complete discharge information under laboratory conditions, which is more convenient to carry out the research work on the discharge mechanism, and the comparability between the defect model and the actual defect in the electric field distribution characteristics and discharge mechanism is fully considered.

Two sets of joints test samples with the metal defects were made, and the area, size and density of the 2 sets of defective copper sheets were basically consistent. After the experiment, the same method is used to analyze the partial discharge data. It is found that the initial discharge voltage, the breakdown voltage and the duration of the partial discharge are different in value, but the overall development trend and the change rule of the partial discharge are basically the same. This paper focuses on the development process of PD from the initiation of the defect to the whole process of breakdown, and in order not to be so wordy, one of the typical experimental results are analyzed in depth. 


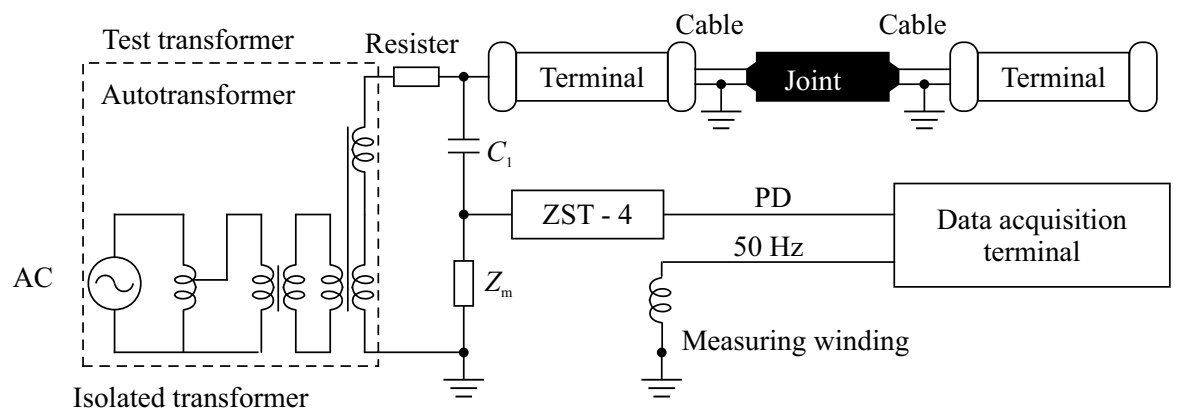

Fig. 2. Diagram of the experimental circuit

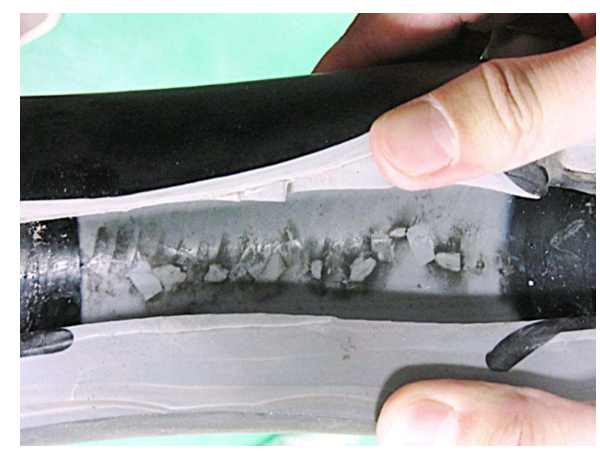

Fig. 3. Disintegration photo of the test sample with discharge marks on the silicon rubber surface

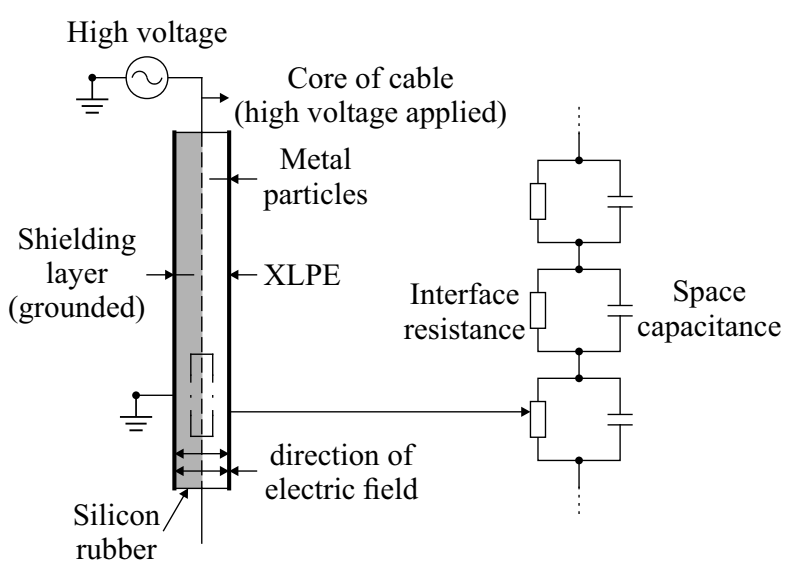

Fig. 4. Equivalent circuit of the silicone rubber/XLPE interface with metal particles

\subsection{Experimental circuit}

To avoid the corona at the endings of the cable, oil cup-typed terminals are assembled, and cables are connected to the test transformer via the terminals. Experimental circuit is shown in Fig. 2, the corona free power system consists of voltage regulator, test transformer of $50 \mathrm{kV}$, protective resistance and coupling capacitor. PD measurement system is parallel connected to the sampling impedance. A data acquisition card with 2 channels (NI PXI-5114, $125 \mathrm{MHz}$ bandwidth, $250 \mathrm{MS} / \mathrm{s}$ ) is used to obtain the PD signal in $20 \mathrm{~ms}$, meanwhile, the phase of the applied voltage is obtained via the transformer measuring winding.
Withstand voltage test in $45 \mathrm{kV}$ and $\mathrm{PD}$ test are carried out as shown in Fig. 3. The high voltage is applied at the end of the left oil immersed terminal, and the shielding layer at both ends is extended and linked by the ground wire. The background interference is less than $3 \mathrm{pC}$.

\subsection{Test method}

The applied voltage is raised until stable PD signal is formed, and then the step voltage is applied to accelerate the deterioration of insulating. Once the insulation is badly degraded, the applied voltage is kept in constant. The IVPD of the defect is $8 \mathrm{kV}$, the voltage is raised up to $40 \mathrm{kV}$ with step of $4 \mathrm{kV}$, and is held in 2 hours in each step. The discharge intensity is obviously increased when the voltage is $40 \mathrm{kV}$, therefore the voltage is decreased to $38 \mathrm{kV}$ after which is held in 2 hours. Finally, the breakdown happens after 1.4 hours. The total time of the test is 19.4 hours.

\section{Analysis of test results}

\subsection{Phenomenon of breakdown process}

The amplitude of PD signal increases heavily when the applied voltage is $36 \mathrm{kV}$, and rat-tat sound can be heard in every $2 \mathrm{~min}, 20 \mathrm{~min}$ before breakdown. The test sample is cut open in axial direction, as shown in Fig. 3.

According to Fig. 3, there are black carbon marks distributed between cooper pieces on the surface of XLPE and the interface of silicon rubber. The conductive channels in axial direction are formed and connect the cable core to the outer semi conductive layer.

In normal prefabricated cable joints, solid interface is formed between the XLPE body and rubber. Because the permittivity of two materials is nearly equal, and the applied voltage distributes according to permittivity, the electric field is uniform. Meanwhile, the rubber is elastic and under high pressure, the interfaces are closely contacted, which limits the excitation of conductive particles. In addition, the silicone grease molecules play a role of barrier, which further blocks the excitation of electrons, and leads to the higher breakdown voltage of creeping discharge. 


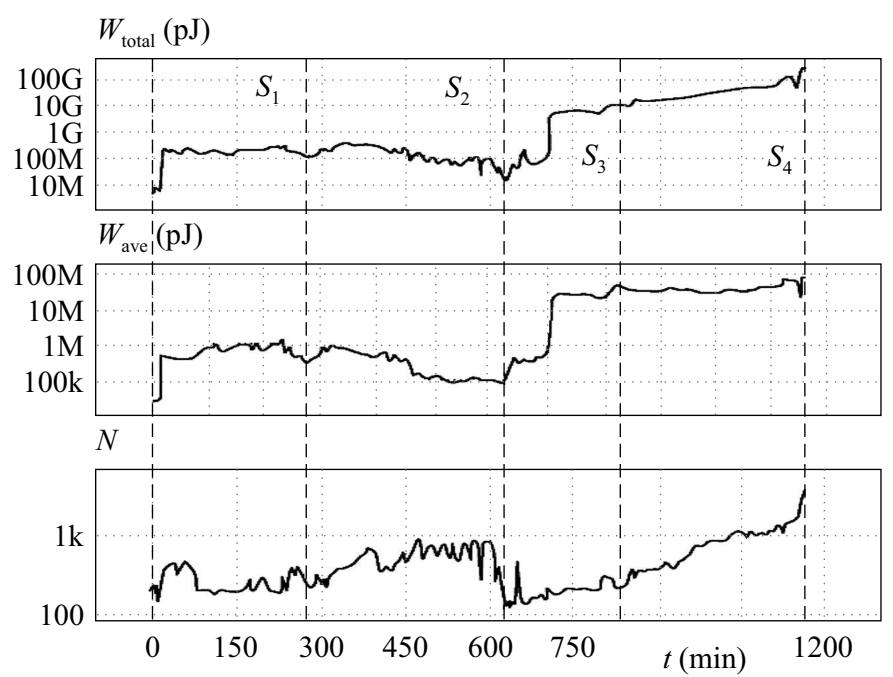

Fig. 5. Curves of 3 PD characterization parameters

Table 1. Duration and Voltage of Each Stage

\begin{tabular}{lcc}
\hline Stage & Duration $/$ min & Applied Voltage $/ \mathrm{kV}$ \\
\hline I & $0 \sim 270$ & $8 / 12 / 16$ \\
II & $270 \sim 625$ & $16 / 20 / 24 / 28$ \\
III & $625 \sim 830$ & $28 / 32$ \\
IV & $830 \sim 1164$ & $32 / 36 / 40 / 38$ \\
\hline
\end{tabular}

However, if the metal particles locate on the interface, the electric field is changed obviously, which destroys the insulation. For the convenience of analysis, the defect is abstracted as a circuit network, where each electric unit is composed of the interface resistance and the capacitor of particle and silicone grease, as shown in Fig. 4.

The space capacitance is considered as the capacitance formed by the metal particles and the silicone grease between the particles. According to the equivalent circuit, floating potential is produced on the particles when applied voltage increases. Then the electric field on the interface is distorted, and the resistance decreases, the voltage on the capacitor becomes larger. With the increasing of the applied voltage, large amount conductive particles are produced via the broken-down capacitor which excites the creeping discharge on the ending at first. By the combined effect of high temperature of PD and the bombardment of particles, carbide is separated out from the capacitor, namely the carbonized conducting channels are formed. With the extension of the channels, the electrode gap constantly decreases, which increases the voltage on the space capacitor, and the capacitors are broken down one by one, meanwhile, discontinuous arc appears at the ending of the channel combined with rattat sound. Once the electrodes are connected by the channel, the interface of XLPE and silicon rubber is totally broken down, and the combined channels are shown in Fig. 3.

\subsection{Development trend of partial discharge}

According to the test results, the number of PDs $N$, the average energy of PD Wave and the total energy of $\mathrm{PD} W_{\text {total }}$ in each $10 \mathrm{~min}$ are calculated, as shown in Fig. 5.

Three PD characterization parameters increase suddenly in 20 min after the initial of PD which indicates that the partial insulation is destroyed. Wave and $W_{\text {total }}$ are kept as the higher amplitude and become steady in the next $250 \mathrm{~min}$, during which the curve of $N$ oscillates slightly, during which the PD is more stable and the space charge in the interface is increasing slowly, namely the space capacitances are being recharged as the main insulation has not been broken. With the curve of $N$ oscillating more violently, Wave and $W_{\text {total }}$ trend to decrease. 352 min later, curves of Wave and $W_{\text {total }}$ increase in three times but $N$ becomes even less. The average energy and total energy of PD decrease little, but the discharge number decreases fast after oscillation at about $620 \mathrm{~min}$. The fast change of discharge number macroscopically indicates the intensification of insulation deterioration, which should be analyzed via the statistical properties of curves. 204 min later, the curves of energy become steady again, Wave slightly decreases while $N$ increases and changes faster than energy. Finally, the test sample is broken down in the last $240 \mathrm{~min}$. The development stages of PD are divided based on the inflection points in the curves, whose duration and applied voltage is shown in Tab. 1.

\section{Statistical characteristics of partial discharge}

The spectrums of $\varphi-n$ and $\varphi-q_{\text {ave }}$ and grayscale maps are built for each stage. Spectrums of $\varphi-n$ and $\varphi-q_{\text {ave }}$ indicate repetition rate and average energy of PD in phase distribution respectively, the phase of applied voltage is divided into 128 intervals. Grayscale maps indicate the 

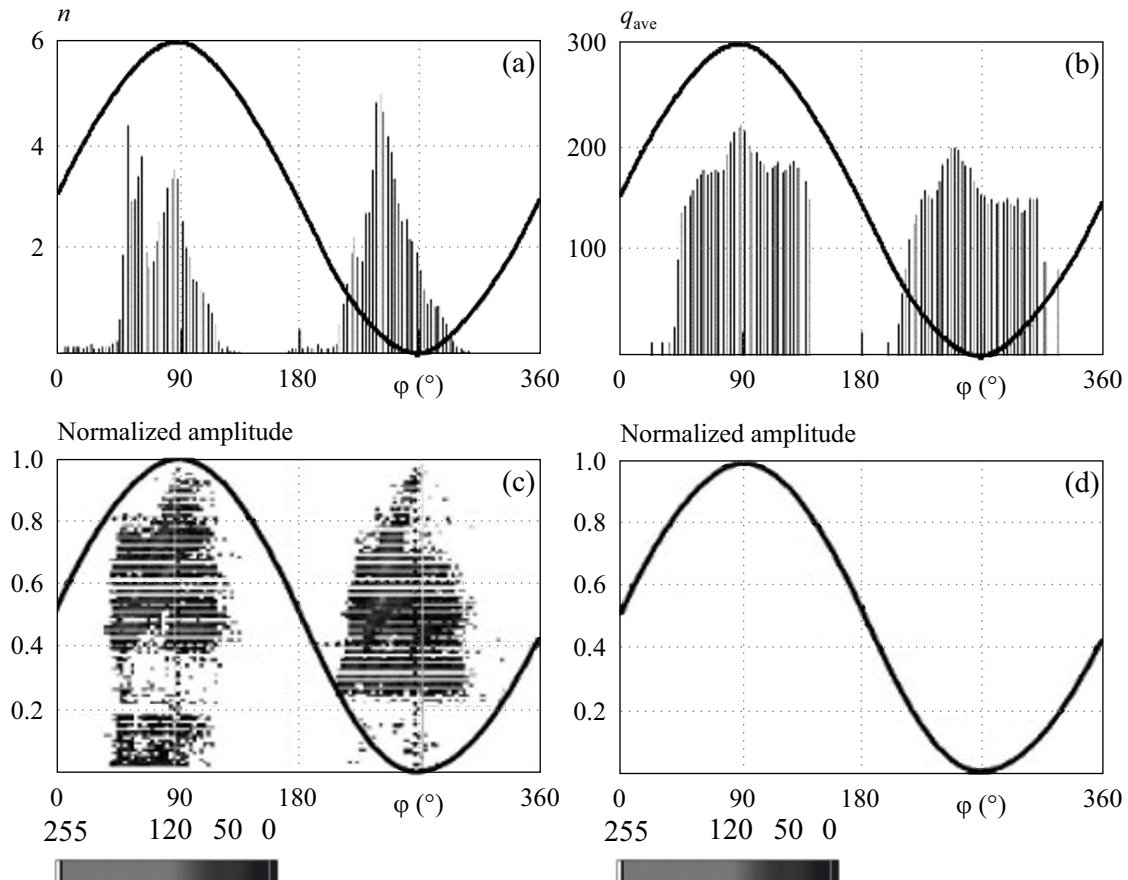

Fig. 6. Phase characteristics spectrograms at stage I: (a) $-\varphi$-n spectrum, (b) $-\varphi$ - $q_{\text {ave }}$ spectrum, (c) - range normalized grayscale map, (d) - maximum normalized grayscale map
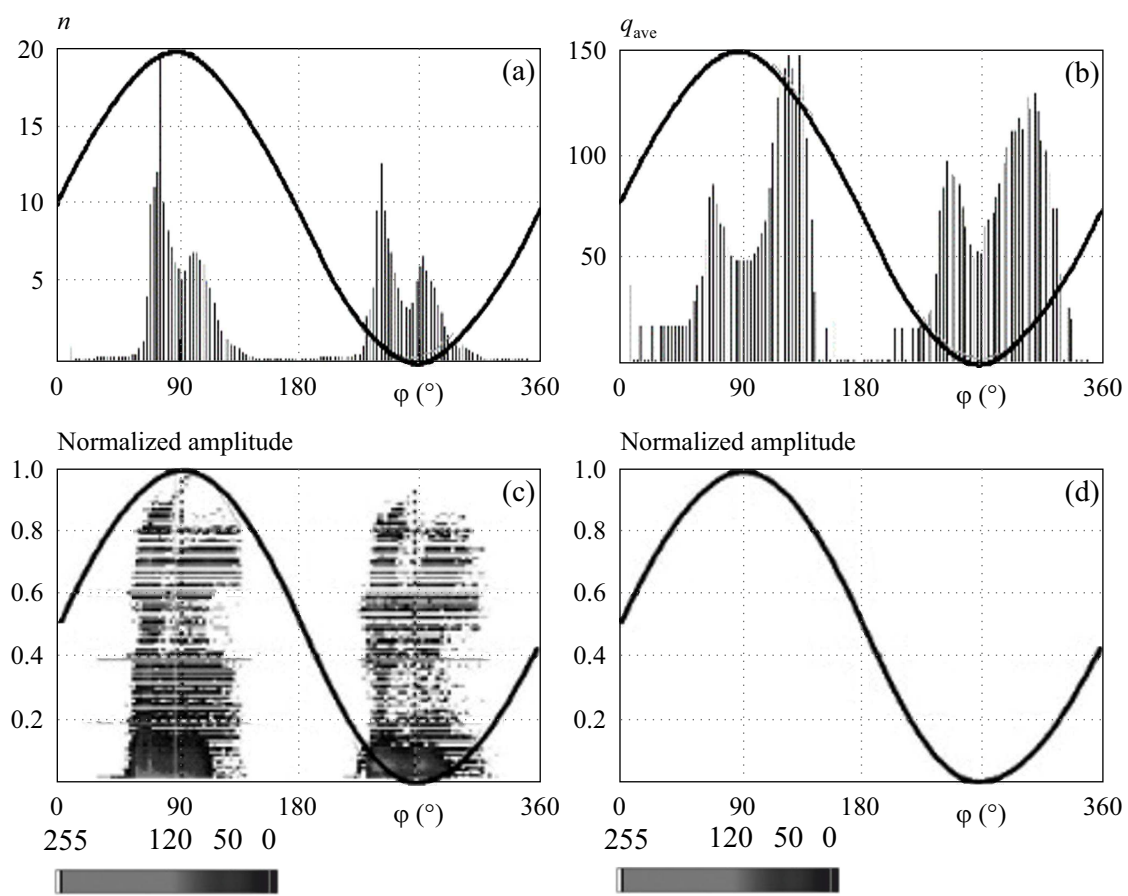

Fig. 7. Phase characteristics spectrograms at stage II: (a) $-\varphi-n$ spectrum, (b) $-\varphi$ - $q_{\text {ave }}$ spectrum, (c) - range normalized grayscale map, (d) - maximum normalized grayscale map

repetition rate and discharge energy in phase distribution. The discharge quantity $q$ is normalized as $q_{\text {norm }}$, then $\varphi-n$ and $\varphi-q_{\text {norm }}$ plane is divided in to $128 \times 128$ areas, repetition rate in each area is calculated. Meanwhile, grayscale maps are built in both range normalization and maximum normalization to describe the details and compare the features of each stage respectively.

\subsection{Characteristics of stage I}

The spectrums and grayscale maps of stage I are shown in Fig. 6. PDs are reflected between $38^{\circ}-140^{\circ}$ and $207^{\circ}$ $311^{\circ}$. The repetition rate in the negative half-cycle is larger, and the average energy in two half-cycles are nearly the same. Characteristics turn to be maximum near the peak of the applied voltage. In phase domain, 

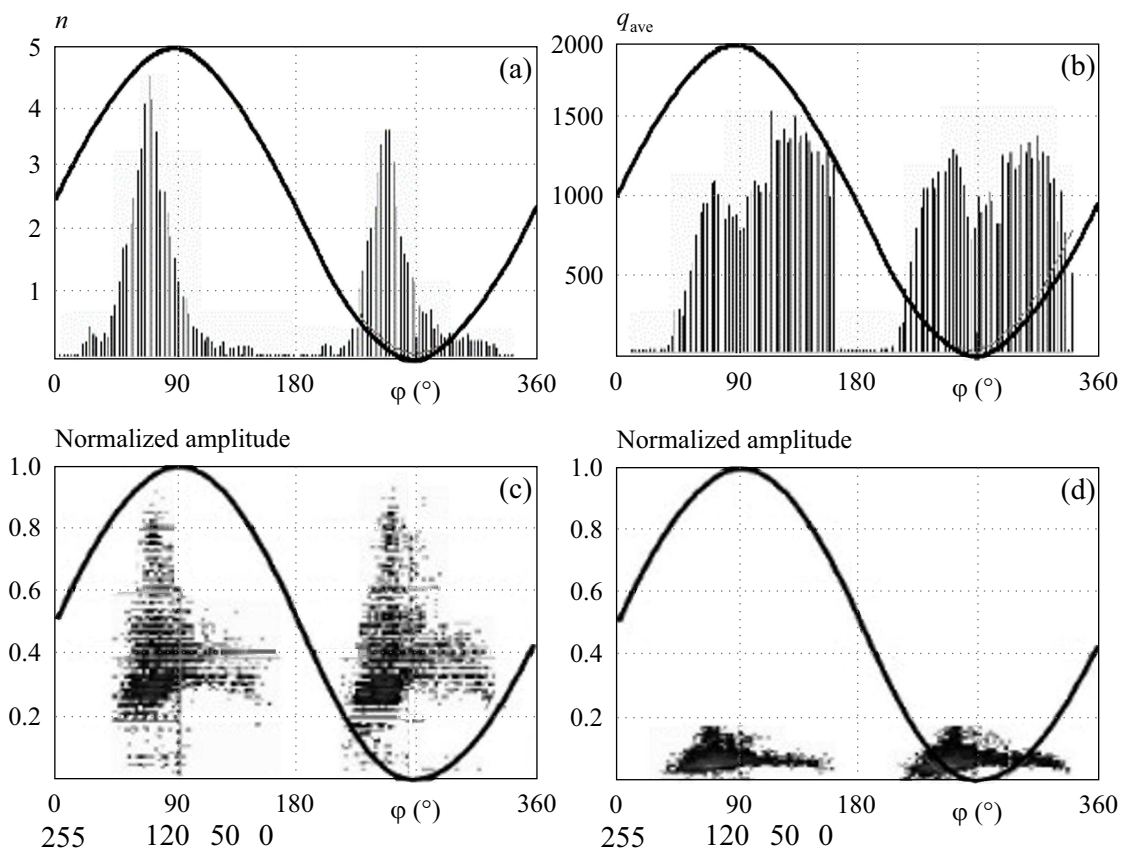

Fig. 8. Phase characteristics spectrograms at stage III: (a) $-\varphi$ - $n$ spectrum, (b) $-\varphi$ - $q_{\text {ave }}$ spectrum, (c) - range normalized grayscale map, (d) - maximum normalized grayscale map
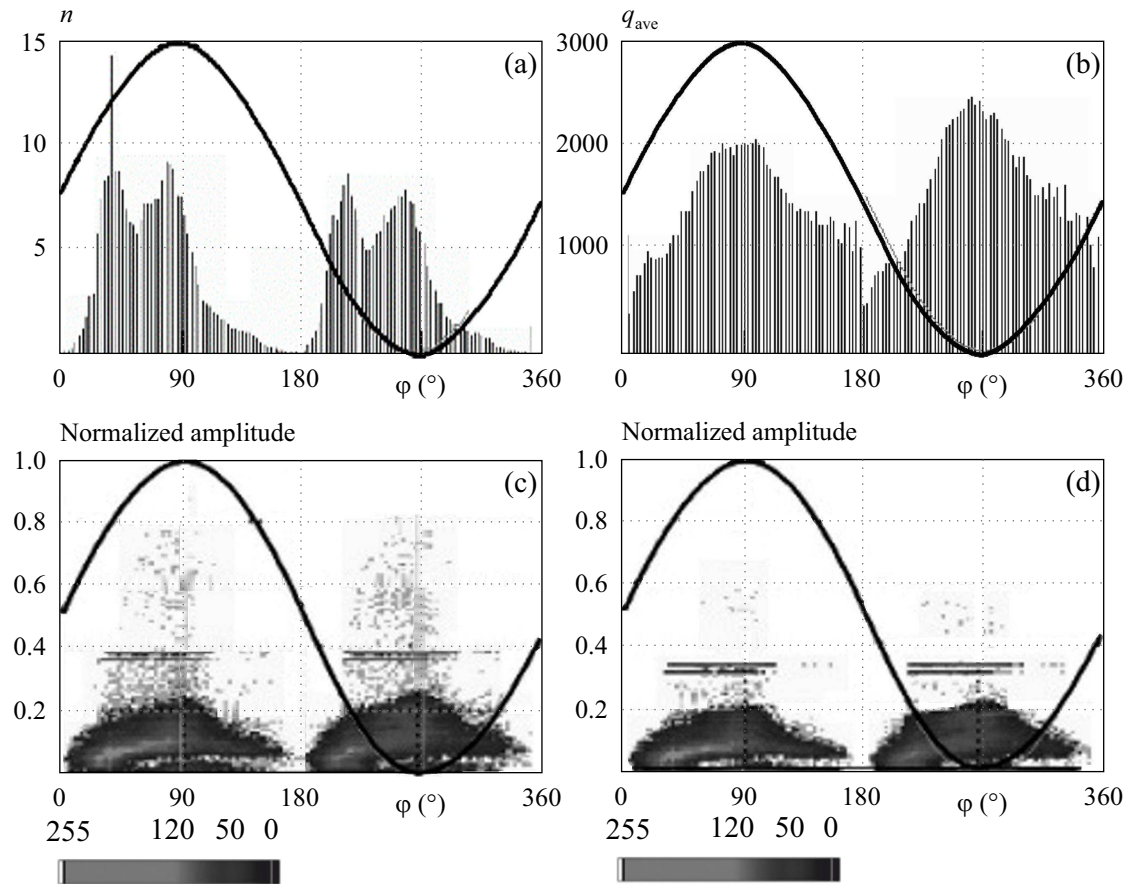

Fig. 9. Phase characteristics spectrograms at stage IV: (a) $-\varphi-n$ spectrum, (b) $-\varphi-q_{\text {ave }}$ spectrum, (c) - range normalized grayscale map, (d) - maximum normalized grayscale map

the envelope of $N$ is formed as double and single peak respectively in each half cycle, and the envelope of $q_{\text {ave }}$ is formed as wavy in both half-cycles. According to range normalized grayscale maps, the discharge area is separated into 2 parts in the positive half-cycle, the density in higher amplitude area is larger, however the discharge area in negative half-cycle is single and uniform. In addition, the discharge values are too small and close in a very narrow area of the maximum normalized grayscale map, which can not achieve characteristic resolution.

\subsection{Characteristics of stage II}

The spectrums and grayscale maps of stage II are shown in Fig. 7. According to the spectrums, PDs are reflected between $30^{\circ}-146^{\circ}$ and $205^{\circ}-337^{\circ}$ which are wider than stage I. In phase domain, the envelopes of 


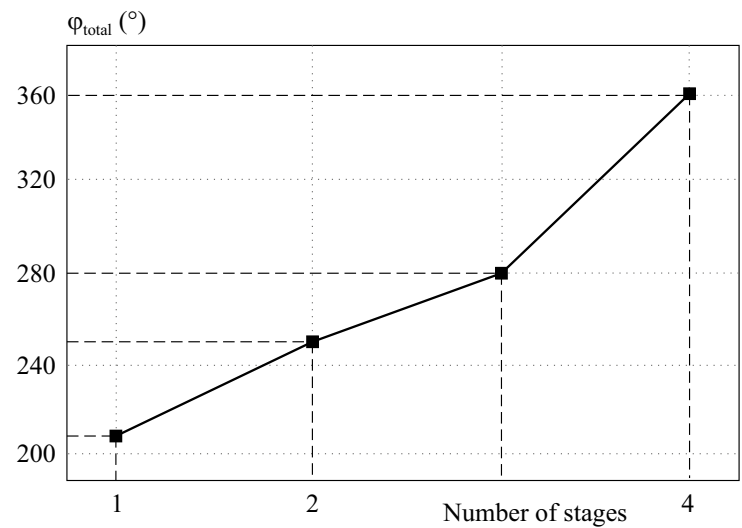

Fig. 10. Trend of PD phase width from stage I to stage IV

$N$ in 2 cycles are formed as double peaks, the repetition rate and average energy are larger in positive half-cycle. According to the range normalized grayscale maps, the density is larger while the amplitude is lower than 0.2 in positive half-cycle, and is very large in negative halfcycle. There is a sunken on the top of the negative cycle area, and is symmetrical about the vertical at $270^{\circ}$. In addition, maximum normalized grayscale map is unable to evaluate defects.

\subsection{Characteristics of stage III}

The spectrums and grayscale maps of stage III are shown in Fig. 8. According to the spectrums, PDs are reflected between $19^{\circ}-162^{\circ}$ and $202^{\circ}-338^{\circ}$ whose width is nearly same with stage II. Compared with stage II, there are two single-peak in each half-cycle in phase domain, the density is very low while the amplitude is lower than 0.2 in the range normalized map, and discharge values are obviously increased in maximum normalized grayscale map.

\subsection{Characteristics of stage $I V$}

The spectrums and grayscale maps of stage IV are shown in Fig. 9. According to the spectrums, PD of creeping discharge are reflected in two double-peaks between $0^{\circ}-360^{\circ}$ and repetition rate becomes larger when the applied voltage is raising. The spectrums of average energy in positive half-cycle is smaller, and is not as sharp as what in the first three stages. Two grayscale maps are similar in structure, and are dense while the amplitude is lower than 0.2 combined with a transverse crack. In the area whose amplitude is larger than 0.2 , discharge points are distributed as triangle and symmetrical distributed along the vertical at $180^{\circ}$. Compared with stage III, points are distributed in a larger area with the increasing of phase width, and the grayscale maps of stage III are considered as the subsets of what in stage IV.

\section{Extraction of characteristics of PD development process}

\subsection{Phase width of $P D$}

According to the stages divided in section 4, the phase intervals are selected while qave is larger than $10 \mathrm{pC}$, which are shown in Tab. 2. The total phase width of PD $\left(\varphi_{\text {total }}^{\circ}\right)$ in each stage is calculated as shown in Fig. 10.

\subsection{Cross-correlation coefficient of PD characteristics}

The coefficient $c c$ indicates the similar degree of the spectrums' shapes, namely $c c$ approximates to 1 when the structures of Figs. are much more similar. The coefficient of spectrograms' envelopes in each stage is calculated by (1), and is shown in Fig. 11.

$$
c c=\frac{\sum_{i=1}^{W} V_{i}^{+} V_{i}^{-}-\left(\sum_{i=1}^{W} V_{i}^{+} \sum_{i=1}^{W} V_{i}^{-}\right) / W}{\sqrt{\left[\sum_{i=1}^{W}\left(V_{i}^{+}\right)^{2}-\left(\sum_{i=1}^{W} V_{i}^{+}\right)^{2} / W\right]\left[\sum_{i=1}^{W}\left(V_{i}^{-}\right)^{2}-\left(\sum_{i=1}^{W} V_{i}^{-}\right)^{2} / W\right]}}
$$

where $V_{i}^{+}$and $V_{i}^{-}$represent the average value of discharge in positive and negative half-cycle respectively.

Figure 11 indicates that cc increases slowly in the first 3 stages bur suddenly rises during stage IV, and approaches to 1 . As the voltage is higher, the deterioration of insulation is more serious, the initial voltage of PD decreases, and PD happens more easily, while the voltage is high enough, polar effect is no longer the main reason of PD, namely the probability of PD is equal in two halfcycles which leads to similar structure and higher cc of the spectrum.

\section{Extraction of characteristics of PD development process evaluation method for its severity}

According to the features in the development of PD, phase width $\varphi_{\text {total }}$ and cross-correlation coefficient cc are employed to describe the process of creeping discharge initiated by metal particles on the silicone rubber/XLPE interface, namely can be used for severity evaluation. The severity of partial discharge is divided into three levels, the initial level, the developing level and the broken level.

The initial level corresponds to the stage I of PD. In this level, insulation is destroyed by the suspended potential on the metal particles. Electric field is huge enough at the edge of particles and finally leads to PD. The statistical characteristics are consistent with the analysis in section 3.1, namely the characteristics suddenly increase and then keep steady. Once the initial level is confirmed, PD monitoring should be strengthened to require the further development and changes.

The developing level corresponds to the stage II and stage III, during which the characteristic values acutely 

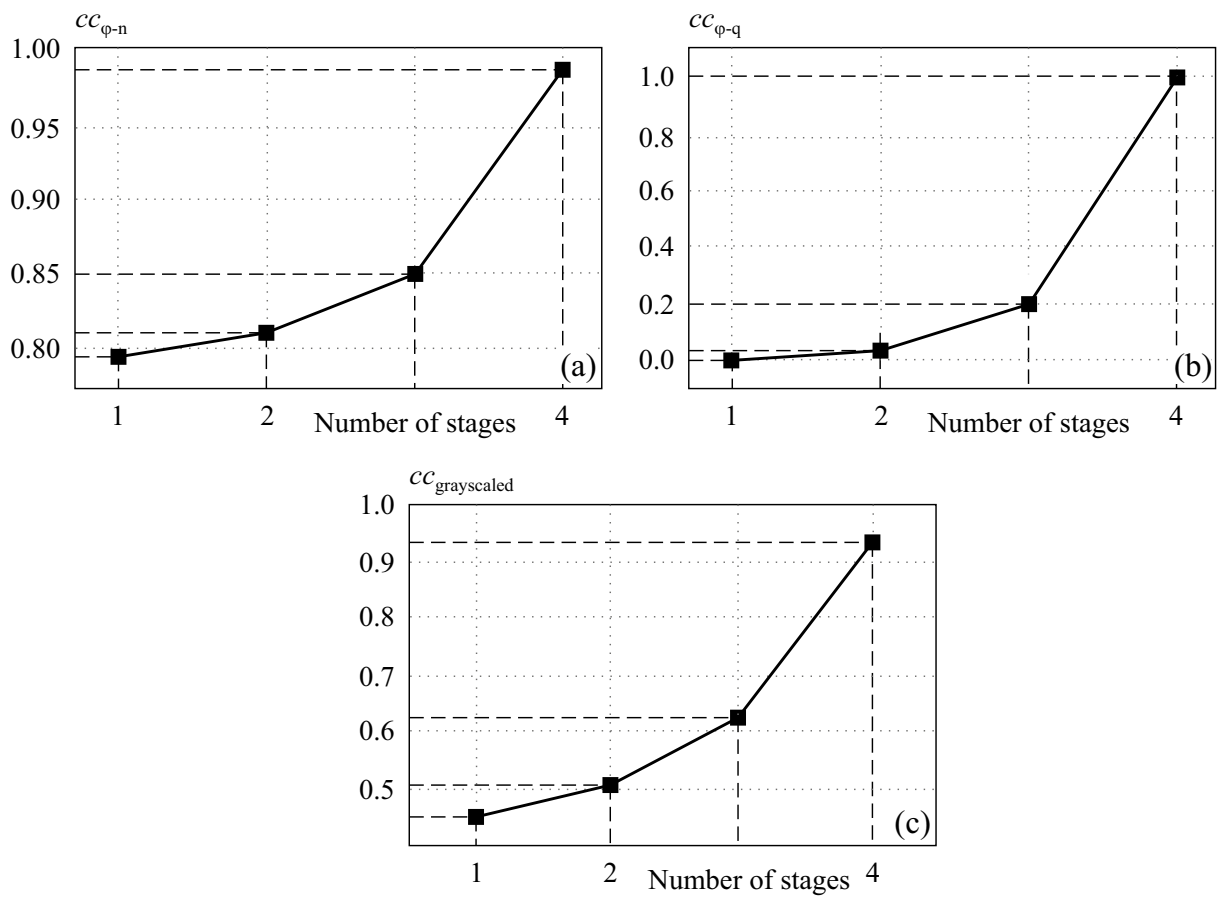

Fig. 11. Trend of cc from each stage: (a) - cc of $\varphi-n$ spectra, (b) - cc of $\varphi-q_{\text {ave }}$ spectra, (c) - of Grayscale Maps

Table 2. Statistics of PD phase width

\begin{tabular}{lcccc}
\hline Stage & Phase Interval $\left(^{\circ}\right)$ & \multicolumn{3}{c}{ Phase Width $\left(^{\circ}\right)$} \\
\hline & Positive Half-cycle & Negative Half-cycle & Positive Half-cycle & Negative Half-cycle \\
I & $38-140$ & $207-311$ & 103 & 105 \\
II & $30-146$ & $205-337$ & 117 & 133 \\
III & $19-162$ & $202-338$ & 144 & 137 \\
IV & $0-180$ & 180 & $180-360$ & 180 \\
\hline
\end{tabular}

change, the phase width increases, and cross-correlation coefficient cc of the spectrums and grayscale maps is raising. With the partial interface insulation breakdown caused by metal particles at the edge, creepage and flashover occur on the interface of silicon rubber and XLPE. Therefore, it is necessary to make appropriate maintenance strategy in advance based on PD monitoring.

The broken level corresponds to the stage IV of creeping discharge. There are no more violent changes in characteristic values, $N$ and $W_{\text {total }}$ almost increase linearly, $N$ and phase width increase obviously faster and inflection points appear in the curves of cc. The space capacitors between the metal particles are broken down one by one which forms carbonization traces and are connected to the electrodes. As the interface is close to broken down, the cable joint should be replaced as quick as possible.

\section{Conclusions}

(1) During the process of creeping discharge caused by metal particles, the number of PDs, average discharge energy and total energy in unit time should be employed to periodically describe the development, and the whole process is divided into 4 stages according to the differences between the spectra.

(2) For the PD caused on the interface of silicon rubber and XLPE, the phase width and cross-correlation coefficients of envelopes in spectrums are increasing in each stage and especially faster in stage III and IV.

(3) Considered with the differences and the trend of spectrums, the evaluation method for the severity of $\mathrm{PD}$ and creeping discharge in cable joints is proposed, which guarantees the operation and maintenance of the power cable.

\section{Acknowledgments}

This work is supported by Science and Technology Project of State Grid Corporation of China (GY71-18040 /5442GY180031).

\section{REFERENCES}

[1] M. Wang, "State Grid Corporation of China's Yearbook" 2011 CA: China Electric Power Press, 2012,, pp. 566. 
[2] M. Wu, B. Ouyang, and W. Li, "Accident Analysis Dynamic Simulation of Smart Substation", Electr, Power, vol. 46, no. 5, pp. 66-70, 2013.

[3] Z. Tang, C. Zhou, W. Jiang et al, "Analysis of Significant Factors on Cable Failure using the Cox Proportional Hazard Model", IEEE Trans. Power Deliv, vol. 29, no. 2, pp. 66-70, 2014.

[4] Z. Tang, W. Zhou, J. Zhao, et al, "Comparison of the Weibull the crowAMSAA Model in Prediction of Early Cable Joint Failures", IEEE Trans. Power Deliv. vol. 30, no. 6, pp. 66-70, 2015.

[5] W. Koltunowicz, L. Badicu, R. Hummel et al, "PD Testing Monitoring of HV Cable Systems", JICABLE'15 9th Int. Conf. Insulated Power Cables, Versailles, France,, pp. 1-6, 2015.

[6] P. Rohwetter, D. Siebler, R. Eisermann, et al, "Progress in Optical PD Detection for Translucent Transparent HV Cable Accessories with Improved Fluorescent Optical Fibres", JICABLE'15 9th Int. Conf., Insulated Power Cables, Versailles, France,, pp. 1-5, 2015.

[7] C. Zhou and G. Chen, "Space Charge AC Electric Breakdown Strength in Polyethylene", 2015 IEEE Conf. Electrical Insulation Dielectric Phenomena (CEIDP) Ann Arbor, MI, pp. 106-109, 2015.

[8] S. Li, Y. Zhu, D. Min, et al, "Space Charge Modulated Electrical Breakdown", Sci. Rep, vol. 6, pp. 1-4, 2016.

[9] D. Wald, J. Perkel, and N. Hampton, "Decision Making \& Forecasting using the Data Available to Utilities - Pitfalls, Challenges", JICABLE'15 9th Int. Conf. Insulated Power Cables, Versailles, France,, pp. 1-6, 2015.

[10] M. Plopeanu, P. V. Notingher, C. Stancu et al, "Underground Power Cable Insulation Electrical Lifetime Estimation Methods", UPB Sci. Bull., vol. 75, no. 1, pp. 223-238, 2013.

[11] S. Tokunaga, T. Tsurusaki, Y. Z. Arief et al, "Partial Discharge Characteristics till Breakdown for XLPE Cable Joint with an Artificial Defect", International Conference on Properties Applications of Dielectric Materials, IEEE, vol. 3, pp. 1206-1209, 2003.

[12] L. Guojun, W. Guopei, X. Jun et al, "Sensitivity Analysis of Cable Oscillating Wave Test System on Multi-Source Defects Diagnostics", CIRED - Open Access Proceedings Journal, vol. 2017, no. 1, pp. 424-427, 2017.

[13] G. Callender, P. L. Lewin, J. A. Hunter, et al, "Modeling Partial Discharge in a Three-Phase Cable Joint Experiment with Minimal Adjustable Parameters", IEEE Transactions on Dielectrics \& Electrical Insulation, vol. 24, no. 1, pp. 279-287, 2017.

[14] X. Zhao, L. Pu, Z. Ju, et al, "Partial Discharge Characteristics Development of Typical XLPE Power Cable Insulation Defects", International Conference on Condition Monitoring Diagnosis, IEEE, pp. 623-626, 2016.

[15] Y. Jiang, H. Min, J. Luo, et al, "Partial Discharge Pattern Characteristic of MV Cable Joints with Artificial Defect", China International Conference on Electricity Distribution, IEEE, pp. 1-3, 2011 .

[16] K. Uchida, T. Hirata, I. Tanaka et al, "Partial Discharge Breakdown Characteristics of Various Defects in XLPE Cable Joints", Ieej Transactions on Power \& Energy, vol. 115, no. 10, pp. 1185-1192, 2008
[17] I. A. Metwally, A. H. Al-badi, A. S. Al-hinai et al, "Influence of Design Parameters Defects on Electric Field Distributions Inside MV Cable Joints", Electrotechnical Conference, IEEE, pp. 1-6, 2016.

[18] G. Li, Z. Luo, J. Xiong et al, "Statistical Characteristics of Partial Discharge Caused by Typical Defects in Cable Joint under Oscillating Voltage", International Conference on Power System Technology, IEEE, pp. 1368-1373, 2014.

19] W. Cao, Y. Zhang, M. A. Huancheng et al, "Analysis of Influences of Defects on Electric Field Distribution of Composite Interface of Cable Joint", Power System \& Clean Energy, 2016.

[20] X. Zhou, J. Cao, S. Wang et al, "Simulation of Electric Field Around Typical Defects in 110 kV XLPE Power Cable Joints", International Conference on Circuits Devices Systems, IEEE, 2017.

Received 12 February 2019

Wen-Zhi Chang was born in Gansu, China, in 1983. He received the $\mathrm{MS}$ and $\mathrm{PhD}$ degrees in electrical engineering from North China Electric Power University (Beijing) in 2009 and 2013, respectively. Currently, he is a Senior Engineer with China Electric Power Research Institute, Beijing, China. His research interests are condition monitoring of power apparatus.

Fei Du was born in Beijing, China, in 1992. He received the MS in electric and engineering from Xi'an Jiao Tong University (Xi'an) in 2017, respectively. Currently, he is an Engineer with China Electric Power Research Institute, Beijing, China. His research interests are condition monitoring of power apparatus.

Jian-Gang Bi was born in Inner Mongolia, China, in 1977. He received the MS in control science and engineering from Xi'an Jiao Tong University (Xi'an) in 2003, respectively. Currently, he is a Professor Level Senior Engineer with China Electric Power Research Institute, Beijing, China. His research interests are condition monitoring of power apparatus.

Shuai Yuan was born in Inner Mongolia, China, in 1980. He received the $\mathrm{PhD}$ degrees in Harbin Institute of Technology in 2009. Currently, he is a Senior Engineer with China Electric Power Research Institute, Beijing, China. His research interests are condition monitoring of power apparatus.

Yan-Peng Gong was born in Hebei, China, in 1983. He received the MS degree in electrical engineering from China Electric Power Research Institute in 2009. Currently, he is a Senior Engineer with China Electric Power Research Institute, Beijing, China. His research interests are condition monitoring of power apparatus.

Yuan Yang was born in Anhui, China, in 1986. He received the MS degree in electrical engineering from North China Electric Power University (Beijing) in 2010. Currently, he is a Senior Engineer with China Electric Power Research Institute, Beijing, China. His research interests are conditionbased diagnosis of power apparatus. 